\title{
Germaine Berton : une criminelle politique éclipsée
}

\section{Fanny Bugnon}

Article publié dans Nouvelles Questions Féministes, "Les logiques patriarcales du militantisme », vol. XXIV, 3, 2005.

L'affaire Germaine Berton débute le 22 janvier 1923 lorsque la militante anarchiste, alors âgée de vingt ans, abat à bout portant Marius Plateau', secrétaire général de la Ligue d'Action française et chef des Camelots du Roi, dans les locaux parisiens du mouvement nationaliste. A l'issue d'un procès entamé le 18 décembre 1923 et émaillé d'incidents quotidiens, cette affaire se conclut le 24 décembre avec l'acquittement, par la Cour d'assises de Paris, de Germaine Berton.

Parce que les deux protagonistes ont des positions politiques diamétralement opposées, parce qu'il s'agit d'une femme qui tue un homme, parce qu'elle agit seule et revendique le caractère politique de son geste, la résonance de cet acte en fait l'un des événements majeurs de l'année 1923 en France.

L'affaire Germaine Berton voit le jour dans un climat de tensions violentes, tant sur le plan national qu'extérieur. Le Bloc national de Poincaré est alors l'artisan d'une politique conservatrice, marquée notamment par un nationalisme agressif et la répression du mouvement ouvrier communiste. Cette politique peut être envisagée comme le reflet des angoisses d'une société hantée par les ravages de quatre années de guerre. Les rapports sociaux de sexe ne sont pas exempts de ces tensions, loin s'en faut. Les relations entre femmes et hommes s'articulent dans un contexte de définition rigide des identités et des rôles, de réaffirmation stricte du féminin et du masculin. Tout écart est mis à l'index et accusé d'altérer l'état de la nation. Les propos d'un militaire pamphlétaire résument à leur façon cet état d'esprit :

"Je veux indiquer que tout le mal moral résulte dans les décompositions sociales, aux heures des civilisations raffinées, du fait même que la femme se déprave, perd son caractère naturel, croit s'émanciper en s'affublant du carcan masculin. Il ne faut pas que l'homme passe de l'activité à la passivité, et que la femme de la passivité passe à l'action". "

En ce sens, les positions nationalistes se présentent comme garantes de l'ordre social et sexué. Comme le souligne Erika Apfelbaum (2004:46) à propos de la conception naturaliste de l'ordre social, «stigmatiser au nom d'un ensemble de représentations et de normes qui se donnent comme des vérités « naturelles » et universelles pour mieux particulariser, et surtout exclure de l'espace social où s'élaborent les décisions concernant le contrat social font partie de l'arsenal des modalités de la domination ».

\footnotetext{
${ }^{1}$ La cible initiale de Germaine Berton était Léon Daudet. Ne parvenant pas à l'atteindre, elle s'est rabattue sur Marius Plateau.

${ }^{2}$ Grandjean, Georges, De la dépravation... des femmes... des décadences !, Paris : Maison d'art et d'édition, 1919, p.16-17.
} 
L'Action française connaît son apogée dans l'après-guerre, avec près de 50000 numéros quotidiens. Au début de l'année 1923, les royalistes pressent Poincaré d'abandonner la difficile majorité du Bloc national pour s'allier avec la droite nationaliste. Les Camelots du Roi, quant à eux, sont une organisation de combat royaliste, de type paramilitaire, composée en majorité d'étudiants. Marius Plateau, proche collaborateur de Léon Daudet, constitue donc un personnage central, à la fois secrétaire général de la Ligue d'Action française et responsable des forces supplétives que sont les Camelots du Roi.

Le mouvement anarchiste français, de son côté, autrement plus marginal, est essentiellement investi sur le terrain des luttes sociales. Germaine Berton, pour sa part, n'est pas particulièrement connue sur la scène politique. Elle a bien écrit quelques articles dans des feuilles anarchistes, mais cela demeure confidentiel. Son geste rappelle les attentats anarchistes de la fin du $\mathrm{XIX}^{\mathrm{e}}$ siècle, mais Eugen Weber (1985) tempère la pertinence de cette angoisse :

"Les anarchistes de 1920 avaient peu de chose en commun avec leurs prédécesseurs, les lanceurs de bombes de jadis; ils étaient individualistes, pacifistes, anti-autoritaires, et sans le sou. Leurs principaux ennemis ne se trouvaient plus autant parmi les bourgeois qu'aux extrêmes de la société bourgeoise : les nationalistes agressifs dont les royalistes étaient le symbole le plus militant » (192).

En outre, Germaine Berton rassemble les différentes caractéristiques des figures qui hantent l'imaginaire social : elle a vingt ans, elle est célibataire, elle a quitté sa mère en province pour Paris, elle ne travaille pas, a déjà été condamnée pour port d'arme prohibée et outrage à agent, et milite au sein de la mouvance anarchiste parisienne, rassemblée autour du journal Le Libertaire. Elle est donc de cette frange de la société en marge, en rupture, qui rejette les valeurs traditionalistes de l'époque.

Ce qui est construit et érigé en " affaire » au cours de l'année 1923 constitue un cas de violence mise au service d'un idéal politique. Acte radical d'une femme marginale par ses opinions politiques, le geste de Germaine Berton jette le trouble dans la hiérarchie des normes criminelles et sociales de l'époque. Le meurtre de Marius Plateau choque d'autant plus que son assassin est une femme. «Les femmes criminelles apparaissent d'autant plus inquiétantes qu'elles sont rares, comme si leur faible présence était inversement proportionnelle à leur dangerosité » commente Frédéric Chauvaud (2002 : 276). En ce qui concerne Germaine Berton, le caractère politique de son crime la singularise au regard de la criminalité habituellement considérée comme féminine (vol, infanticide, crime passionnel...), et la presse d'information le souligne :

"Un crime célèbre, une femme, une jeune fille de vingt ans au banc des accusés et non pas pour un de ces crimes passionnels qui arment de couteaux ou de revolvers de jolies mains trop promptes, mais pour un crime politique, un crime d'idées, voilà du nouveau, n'est-ce pas ? " (Le Petit Parisien, cité dans L 20.12.1923).

\footnotetext{
${ }^{3}$ Pour les références : $L$ pour Le Libertaire et AF pour L'Action française.
} 
Le traitement médiatique de l'affaire Germaine Berton par les deux journaux militants directement impliqués sera étudié ici : à travers Le Libertaire, hebdomadaire puis quotidien anarchiste, et L'Action française, sous-titrée "organe du nationalisme intégral », dirigée par Léon Daudet et Charles Maurras, publication du mouvement politique du même nom. L'observation du traitement médiatique par deux journaux militants profondément antagonistes vise à déterminer les enjeux d'une telle affaire et la dimension particulièrement symptomatique, en terme de production et de renforcement de genre, des représentations sociales, morales et politiques des femmes. En nous intéressant à la réception de l'affaire Germaine Berton, nous entendons démontrer dans quelle mesure le genre participe de la construction des discours politiques et répressifs.

\section{Sexisme en regards croisés}

Évoquant le cas des femmes révolutionnaires russes de la fin du XIXe siècle, Marie-Claude BurnetVigniel (1990) affirme l'importance de la dimension sexuée dans la considération de la violence politique, expliquant que « la militante encourait certainement un blâme social plus lourd que l'homme, comme si s'ajoutait, dans son cas, au délit politique qui justifiait seul la répression contre ses compagnons, un crime de dénaturation de la femme »(15). Les journalistes se penchent ainsi longuement sur la vie de la militante anarchiste, disséquant les moindres aspects de son existence.

Se dessinent alors deux portraits de Germaine Berton dont les propos relaient les théories sur la "nature féminine », les anarchistes comme les nationalistes fondant leurs discours sur ce genre de stéréotypes conservateurs. Incarnation de la féminité idéale, la femme n'existe qu'au singulier guidé par la «nature »; " une femme n'est jamais qu'une femme, un objet interchangeable sans autre caractéristique que la féminité, dont le caractère fondamental est d'appartenir à la classe des femmes » écrit Colette Guillaumin (1992: 15). L'analyse des discours concernant Germaine Berton nous invite à suivre cette critique. En effet, les représentations médiatiques de la militante anarchiste se font l'écho des stéréotypes féminins élémentaires qui considèrent les femmes comme soumises, douces et sensibles par « nature », une « nature » qui fixe les règles sociales.

Si Germaine Berton a franchi des limites sociales, il semble que l'on cherche, malgré tout, à lui conserver une part de la dimension « naturelle » attribuée aux femmes. George Mosse (1997) insiste sur un point important pour notre analyse : «les stéréotypes sont par nature des images publiques. C'est parce qu'ils font de l'invisible quelque chose de visible et de public qu'ils acquièrent leur importance sociale et politique » (13). Ces stéréotypes font apparemment fi des frontières politiques, qualifiant d'évidence des comportements normés, ils dispensent d'y réfléchir. "Fière et douce » (L 19.12.1923), "délicate et sensible, Germaine Berton s'est toujours conduite en bonne camarade » (cité par Le Temps, 22.12.1923) explique Lecoin, rédacteur du Libertaire, lors de son audition devant la Cour d'assises. Les partisans de Germaine Berton reprennent ainsi à leur compte les stéréotypes féminins, signifiant que, malgré sa marginalité politique, elle n'en est pas moins et surtout une femme. 
La militante anarchiste est avant tout envisagée dans sa différence sexuelle, présentée par ses soutiens comme une faible créature lâchement attaquée. Parce que la criminologie propose une interprétation naturaliste dans laquelle la violence, constitutive de l'identité masculine, de la virilité, est opposée à la délicatesse et à la passivité, présentées comme des caractères féminins, elle induit le renforcement des schémas sexistes de l'époque. L'objectif poursuivi par Le Libertaire et les autres soutiens de Germaine Berton est de rattacher la militante anarchiste à la catégorie « femme » malgré son crime, véritable transgression du code de bonne conduite féminin. Et ce, non pas en considérant que les femmes puissent être violentes, mais en affirmant leur incapacité à se défendre : « tu es leur victime, et nous ne permettrons pas qu'ils s'acharnent plus longtemps sur toi » ( $\mathrm{L}$ 30.03.1923). Enfermer Germaine Berton dans cette dimension est un moyen d'atténuer le sens de l'assassinat de Marius Plateau et de rechercher la compréhension de la Cour pour celle que ses partisans présentent comme une victime. Un des aspects délibérément exploités est celui de la figure maternelle absente. La mère supposée indigne est mise en accusation pour atténuer la responsabilité du geste meurtrier de Germaine Berton. Le témoignage de Séverine, dernière témoin de la défense, est à ce titre éloquent : " mais où est donc la mère de cette enfant, cette mère sait que sa petite est là, sur le banc, et, qui n'est pas à son côté... Ah! La voilà, l'excuse de cette enfant!" (cité par Le Temps, 24.12.1923). Cette référence est à inscrire dans le contexte particulier des années qui font suite à la Grande Guerre. En effet, les années vingt sont l'occasion d'une réaffirmation des valeurs morales qui régissent l'ordre social sexué. Valeur positive par excellence, la maternité constitue le point de référence de l'ensemble des fonctions assignées aux femmes. Par glissement, les partisans de Germaine Berton tendent à son infantilisation, la jeunesse étant présentée par certains comme une excuse, ce qui a pour effet de relativiser sa réelle responsabilité et sa crédibilité politique. En ce sens, on peut relever une série de termes récurrents : Germaine Berton est « une gosse de vingt ans » ( $\mathrm{L}$ 26.01.1923), « une noble jeune fille blâmable et émouvante » (L 27.04.1923), « une petite jeune fille » ( $L$ 18.12.1923). Germaine Berton ne semble donc pouvoir échapper à sa condition de femme, en proie à une faiblesse, à une fragilité sur laquelle le journal anarchiste insiste lourdement. Cet usage délibéré de stéréotypes, autant dans Le Libertaire que par $\mathrm{M}^{\mathrm{e}}$ Torrès, son avocat, entre cependant en contradiction avec les déclarations de Germaine Berton qui refuse de se présenter comme une victime de qui que se soit. Cette contradiction notoire brouille les pistes tout au long de l'affaire.

«Les mœurs désorganisées et les actes mauvais ne préparent que trop bien à l'acceptation des doctrines de désorganisation et d'indiscipline et celles-ci à leur tour favorisent l'extension des mauvaises mœurs et des actes pervers ; cette réciprocité de services accélère toujours la vitesse du mouvement qui nous emporte » écrit en 1920 Paul Bureau, pamphlétaire réactionnaire de son état" L'Action française se penche, en ce sens, sur la moralité de Germaine Berton. C'est sur ce terrain que va être travaillée la question de la responsabilité de la militante anarchiste, tant par l'accusation que par la presse de droite, et ce en écho au portrait de la jeune fille fragile dressé par ses propres soutiens. Les nationalistes se livrent à une critique de la fragilité de l'accusée. Pour eux, il s'agit plutôt d'une odieuse stratégie de la défense qui vise à atténuer ses responsabilités dans l'assassinat de

\footnotetext{
${ }^{4}$ Paul Bureau, L'indiscipline des mœurs, Paris : Bould\&Gay, 1920, p.169-170.
} 
Marius Plateau. A partir de cette question se construit un discours global qui s'efforce de creuser la vie privée de Germaine Berton. "Vue par ses ennemis, la femme révolutionnaire apparaissait d'emblée, par contraste avec la norme sociale, comme suspecte d'immoralité. Elle ne pouvait prétendre au titre d'honnête femme ». Cette analyse de Marie-Claude Burnet-Vigniel (1990: 15) au sujet des représentations des femmes engagées dans le mouvement révolutionnaire russe de la fin du XIX siècle se révèle tout à fait applicable aux discours condamnant Germaine Berton. Cette démarche reflète ainsi les enjeux sociaux qui préoccupent une société obsédée par la réaffirmation d'un ordre social sexué contrarié par les quelques évolutions de la société française de l'époque. Ainsi, de nombreux article insistent sur le caractère dénaturé, voire décadent, des mœurs de Germaine Berton. Serait-elle lesbienne? Aurait-elle avorté ? Collectionnerait-elle les amant·e·s ? Parce que les pratiques sexuelles contrariant un ordre social construit sur l'idée d'un ordre biologique l'hétérosexualité légitime et reproductrice - sont perçues comme une menace, Germaine Berton incarne ce danger profond, ce « relâchement des mœurs ». Du point de vue de ses détracteurs, nous nous trouvons en somme face à un portrait général d'une Germaine Berton dépravée, à la merci des perversions les plus viles. Le traitement est identique à celui des militantes révolutionnaires russes : «l'association des opprobres: révolutionnaire, donc perverse, est devenue un lieu commun, la militante perdant ipso facto, par son engagement, les vertus propres d'une "vraie » femme, la réserve, la pudeur et la chasteté, tandis que les traits de caractère prêtés aux "nihilistes », leur exaltation, leur cruauté et leur fanatisme, deviennent littéralement monstrueux chez elle " (BurnetVigniel, 1990: 16-17). L'Action française propose un portrait général de Germaine Berton par l'intermédiaire de l'un des avocats des parties civiles, $M^{e}$ Campinchi :

«Germaine Berton est un repris de justice (...). C'est une fainéante, une paresseuse, qui préfère, dit l'acte d'accusation, "au lieu de s'assurer par le travail des ressources honorables, subsister misérablement des libéralités incertaines de divers camarades libertaires, dont elle devenait la maîtresse passagère ". C'est une tapeuse : entre un cambriolage et une manifestation anarchiste, elle va taper des religieuses à Nanterre (...). C'est une fille malpropre au moral et au physique (...). C'est une imprudente et une cynique : (...) elle se vante de ses coucheries à deux, parfois même à trois ; elle parle comme d'une chose toute naturelle de s'être fait avorter " (AF 18.12.1923, souligné dans le texte).

En agitant le spectre d'une femme démoniaque, véritable incarnation du "Mal social », ses détracteurs l'intègrent dans la globalité de l'évolution des mœurs contre laquelle ils s'élèvent. Son engagement politique n'est alors considéré que comme la dernière étape de sa dégénérescence morale. Plus encore que son geste criminel, ce sont ses mœurs qui sont condamnables. D'autre part, ce qui se révèle frappant, c'est l'importance accordée aux caractéristiques physiques de l'accusée, conformément aux théories criminologiques. En effet, des descriptions récurrentes s'attachent à démontrer la trahison de la féminité à travers les apparences. II s'agit, par ce genre de procédés, d'affirmer la singularité de la criminelle et de montrer que les traits physiques sont le reflet du caractère moral de l'individue. La perversité supposée de Germaine Berton doit donc nécessairement 
se traduire par des singularités physiques qui la distinguent du mythe de l'éternel féminin. La féminité est en effet, en partie, une question d'apparence. « Est-ce une femme ? » titre ainsi L'Action française (19.12.1923) pour l'ouverture du procès. Certains journaux se plaisent ainsi à disserter sur l'allure masculine de Germaine Berton. Ses cheveux courts et sa lavallière constituent des attributs symboliques de la femme émancipée des années vingt, la garçonne. Comme le soulignent Christine Bard et Françoise Thébaud (1999), « symbole de la modernité des années 1920, la garçonne est une figure négative, l'antithèse de la femme nouvelle dont rêvent les féministes, mais l'exact opposé aussi de l'image traditionnelle de la féminité » (162). Parce que la garçonne constitue une image de rupture avec l'identité féminine traditionnelle - à laquelle la majorité des féministes de l'époque sont attachées -, d'émancipation concrète, Germaine Berton fournit ainsi, aux tenants de l'ordre social, l'occasion de faire le lien entre la criminalité et l'émancipation féminine, bien que la garçonne soit un phénomène relativement marginal et urbain. Germaine Berton n'incarne pas la féminité normée. Pour L'Action française, il y a quelque chose de bestial chez elle : elle est « irascible, méchante et querelleuse comme une teigne » (21.12.1923). Le portrait peut être beaucoup plus dur :

"Cependant que la voix tendre de Mme Séverine déroule la tirade idyllique, au banc des assassins, Germaine Berton baisse ses prunelles révélatrices, ses prunelles de hyène qui se sont mirées dans le sang...» (23.12.1923).

Comme la rhétorique développée par L'Action française vise à démontrer en quoi Germaine Berton s'est exclue de la catégorie "femme ", son caractère est minutieusement étudié et on lui prête une série de qualificatifs complètement opposés à la féminité idéalisée. Ainsi, on insiste sur son sang froid, sa violence. Ce qui choque lors de son procès, c'est l'absence de remords, l'insensibilité dont elle fait preuve. Cela nourrit l'idée de sa perfidie :

"La "Vierge noire » avait cherché, pour l'audience publique, à se rajeunir, à paraître " petite fille ". Mais, sous les cheveux à la Ninon, le fard et le Kohl n'avaient pu que souligner à travers son visage les marques précoces du vice » (AF 19.12.1923).

Que Germaine Berton fasse siennes les valeurs traditionnellement masculines - parce que guerrières - relève presque de l'outrage absolu. Aussi les journaux conservateurs s'emploient-ils à l'isoler dans l'anormalité. En ce sens, l'édition de L'Action française du 19 décembre 1923, au lendemain de l'ouverture du procès, propose un florilège de ce qui est alors considéré comme une trahison de «l'essence féminine »:

"Sèche, froide, fausse, elle manie sans amour, sans enthousiasme, les grands mots dont elle fait ses excuses : individualisme, anarchie (...). Vanité de ce qu'elle croit être ses idées et qui ne sont que ses instincts déchaînés, vêtus des réminiscences de tristes lectures (...). Vanité d'être anormale et monstrueuse (...). Paresse qui semble lui avoir interdit l'amour : les débauches de cette fille n'ont été que des coucheries que nul effort de cœur ou d'esprit n'essayait d'embellir ». 
Germaine Berton n'est pas située par rapport à ses compagnons de lutte, partisans d'un renversement de l'ordre social et politique, mais par rapport à son sexe, et ce, aussi bien par ses défenseurs que par ses détracteurs. Les clichés avancés transcendent les clivages politiques; L'Action française comme Le Libertaire basent une partie de leur argumentation sur la question de la féminité de la meurtrière. S'ils partagent tous deux une norme de sexe qui place au cœur de son propos une définition naturaliste de " la » femme, leurs interprétations divergent : pour les premiers, l'explication du crime réside dans le caractère dénaturé de Germaine Berton, quand les seconds insistent sur l'innocence comme caractéristique de la militante parce qu'elle est avant tout une femme. Quoiqu'il en soit, il s'agit ici de représentations fondées par les mécanismes de construction du genre qui renforcent la différence des sexes et le processus de catégorisation. Fragile ou traîtresse, la présentation de Germaine Berton conditionne la perception des enjeux politiques au cœur de l'assassinat de Marius Plateau par une militante anarchiste. Les commentaires de L'Action française et du Libertaire sont révélateurs de l'état des rapports sociaux de sexe dans la France des années vingt et des angoisses que soulève la revendication d'un meurtre politique par une femme.

\section{Rapports sociaux de sexe et interprétation de l'acte}

Les rapports sociaux de sexe viennent brouiller la question des responsabilités et l'interprétation du crime de Germaine Berton. Les stratégies développées par les deux parties sont, par ailleurs, loin d'être exemptes de contradictions.

\section{La thèse d'un complot}

L'Action française s'attache, tout au long de l'année 1923, à développer la thèse d'un vaste complot dont Germaine Berton ne serait qu'une illustration. Le lien est ainsi fait entre l'assassinat de Marius Plateau et les événements contemporains ${ }^{5}$. Des explications détaillées sont données dans les pages du journal qui consacre sa une au sujet pendant une dizaine de jours, puis des articles quotidiens. L'intégralité des éditions de l'année 1923 est le reflet de cette obsession. " Une balle allemande a tué Marius Plateau » (23.01.1923) titre L'Action française au lendemain de l'assassinat du responsable nationaliste. Le complot est présenté comme un crime contre la France organisé à la fois par des ennemis internes (communistes, parlementaires, journalistes, juges, responsables de la Police) et extérieurs (I'Union soviétique, l'Allemagne, les Juifs). Un vibrant « appel aux combattants et aux patriotes » est ainsi lancé dans L'Action française :

\footnotetext{
${ }^{5}$ Notamment l'occupation française de la Ruhr, consécutive au traité de Versailles, et l'arrestation en janvier 1923 d'une vingtaine de responsables communistes.
} 
"Le crime est commis contre la Patrie : c'est le premier acte des germano-bolchevistes contre l'action de la France en Allemagne. II faut empêcher les actes qui sont projetés. Il faut opposer les forces nationales unies aux agents de l'Allemagne, des Soviets et de la finance internationale " (23.01.1923).

Pour les nationalistes français, il n'y a pas de doute possible : celle qu'ils nomment "la femme Berton » (AF 23.01.1923) se trouve au cœur d'une conspiration aux ramifications multiples. II n'est pas concevable qu'un des leurs ait été tué de sang froid par une simple militante anarchiste. Elle a tiré sur Plateau, certes, mais elle ne peut qu'avoir été manipulée. Le meurtre de Marius Plateau ne serait plus alors que la concrétisation des menaces que L'Action française agite depuis des mois et, plus encore, la preuve de la décadence, de l'altération des forces nationales et des dangers d'anéantissement qui menacent la France.

L'initiative du crime est ainsi retirée à Germaine Berton. Comment une femme du peuple aurait-elle pu abattre un responsable de l'organisation nationaliste ? Cette idée est insupportable pour l'Action française qui s'empresse de trouver un mobile afin de conforter ses propres thèses. "Qui a armé la main de Germaine Berton? " (26.01.1923) s'interroge le quotidien nationaliste. II s'agit alors de démontrer que la militante anarchiste n'a été qu'une exécutante : "Toutes les paroles de Germaine Berton montrent qu'elle a été guidée, aiguillée, informée, conseillée " (AF 8.02.1923). La transgression de l'ordre social et sexué serait trop importante à admettre. La violence politique féminine dérange déjà suffisamment pour qu'on reconnaisse à Germaine Berton l'entière maternité de son geste. Cette attitude de déni sera maintenue jusqu'au procès. "Nullement un crime accidentel, dû à la sauvagerie d'une isolée, mais bien le résultat d'une conjuration germano-révolutionnaire » (AF 6.02.1923), telle est est la position défendue par les nationalistes durant l'année 1923, bien qu'aucun élément sérieux ne vienne étayer cette thèse.

\section{Le déni du caractère politique}

Le caractère politique du crime est soumis à l'interprétation du geste, déterminée par les rapports sociaux de sexe. La position de L'Action française va ainsi connaître un revirement au moment du procès. En effet, immédiatement après l'assassinat de Marius Plateau, il est question de " meurtre politique » (AF 27.01.1923), et la presse française dans son ensemble s'accorde sur la qualification du meurtre : " une femme appartenant aux milieux anarchistes, dans un mobile politique de propagande et de vengeance avouée, a assassiné M. Marius Plateau » (Le Temps, 24.01.1923). Mais l'un des avocats des parties civiles, $\mathrm{M}^{\mathrm{e}}$ Campinchi, affirme pourtant dans sa plaidoirie :

"Mais l'acte de Germaine Berton n'est pas un meurtre politique! C'est le résultat logique d'un système, un attentat gratuit, spécifiquement anarchiste, rien d'autre (...)! Vous êtes anarchiste, et vous avez frappé parce que l'anarchie, c'est le désordre. Vous êtes contre la société » (cité par AF 1.01.1924). 
Comment et pourquoi ce revirement? II s'agit pour l'Action française de décrédibiliser la militante anarchiste et de faire passer l'assassinat de Marius Plateau non plus seulement pour un crime antipatriotique, concrétisation du complot contre les tenants de la "vraie France », mais pour un acte de délinquance, un simple crime de droit commun. On peut avancer l'explication qu'un meurtre reconnu comme politique pourrait bénéficier d'une certaine indulgence de la part du jury. La stratégie de l'Action française vise à obtenir une condamnation maximale pour Germaine Berton. En jouant sur deux tableaux, l'anarchiste délinquante et le complot, les nationalistes espèrent ainsi une sanction exemplaire. Ce faisant, le caractère politique de l'engagement pour l'anarchisme est réduit à de la délinquance maquillée de politique. La négation de la dimension politique poursuit un double objectif : renvoyer Germaine Berton à son état de sujet non politique et refuser le rapport de force auquel l'Action française est soumise. Ce déni est révélateur de la méfiance à l'égard des femmes et de la crainte que les initiatives d'une minorité ne viennent renverser l'ordre social. II s'agit donc de réaffirmer l'incapacité des femmes à traiter de politique.

\section{Une large solidarité}

De son côté, Germaine Berton fédère rapidement les solidarités des opposants à la politique du Bloc national. Bien que membre de l'Union anarchiste, mouvement national constitué en 1920, elle rencontre des échos favorables, notamment dans L'Humanité, L'Oeuvre (quotidien radicalisant de Gustave Théry dont la formule "à gauche, mais pas dans les brancards " a marqué) et L'Ere nouvelle (petit journal radical). Les témoins cités par la défense saluent, parfois avec des réserves, le geste de Germaine Berton. Cette situation originale mérite d'être analysée. En effet, la dimension éminemment politique de l'assassinat de Marius Plateau ne fait pas de doute chez les opposants de l'Action française et c'est en cela qu'elle rassemble. Les discours de soutien vont cependant brouiller eux aussi l'interprétation du crime en tenant des propos contradictoires. Les observations faites au sujet du soutien aux militantes révolutionnaires russes peuvent être transposées à la réaction de solidarité suscitée par la militante anarchiste :

"Les révolutionnaires ne doivent en aucun cas apparaître comme des "laissées pour compte ", mais bien au contraire comme des être exceptionnellement doués à tous égards, que seul leur sens moral détourne d'une vie de privilégiées. Ainsi, le mécanisme de l'accusation n'est pas démonté, ni rendu inefficace ; il n'est qu'escamoté, puisqu'un autre manichéisme se substitue à celui des censeurs et des juges, opposant à l'image de perversité celle d'un ange, d'une sainte entièrement vouée à sa cause, d'une martyre capable d'affronter pour sa foi le châtiment de la mort, un sourire serein aux lèvres » (Burnet-Vigniel, 1990 : 20).

Dans le cas de Germaine Berton, c'est, dans un premier temps, l'esprit de sacrifice et de bravoure qui est salué. L'individualisme, valorisé par certains courants de l'anarchisme, célèbre le culte de la violence efficace. Le Libertaire s'efforce, tout au long de l'année 1923, de louer l'abnégation, l'engagement total de Germaine Berton. Désintéressée, " jeune et jolie, elle aurait pu, comme tant 
d'autres, marchander sa peau, faire de son corps une usine à pognon " (23.02.1923). On loue son courage, l'altruisme absolu dont elle a fait preuve en faisant don de sa vie à la cause : elle a osé atteindre physiquement l'Ennemi. Les formules élogieuses à l'égard de Germaine Berton abondent tout au long de l'année 1923 : «Ô jeune et courageuse amie! Bonne et ardente camarade, comme nous reconnaissons la valeur de ton sacrifice " ( $L$ 16.03.1923), " tu t'es dressée, seule parmi les hommes et les femmes et tu as dit : "Voici ma jeunesse ! Voici ma vie! Je vais mourir pour l'idée !" » (L 3.03.1923).

La solidarité s'affiche donc dans les colonnes du Libertaire afin d'affirmer avec force le soutien de l'ensemble de la mouvance anarchiste à Germaine Berton. L'Union anarchiste publie en ce sens un communiqué au lendemain de l'arrestation de la militante :

"Si nous ne possédons pas le courage d'une Germaine Berton, nous n'aurons point non plus la lâcheté de tous ces chacals de la presse qui piétinent une enfant blessée, dont la prison deviendrait le tombeau si nous ne parvenions à l'en sortir. Non! Qu'on n'attende pas de nous que nous nous désolidarisions d'avec la vaillante jeune fille ! » (L 26.01.1923)

\section{Un double exemple}

$\mathrm{Ne}$ pas abandonner Germaine Berton, c'est aussi en faire un double exemple. Exemple pour l'ensemble des militant·e.s anarchistes et exemple pour les femmes.

Avant tout pour le peuple, comme le montre cet article titré "Du geste individuel à l'action de masse " : "Puisse le geste de Germaine Berton éveiller parmi les exploités un peu de cet héroïsme qui leur manque pour oser - enfin - s'affirmer dans leur vie pratique » (L 2.02.1923).

"La laisserons-nous mourir? Le destin t'avait fait femme. D'aucuns diront que tu étais normalement née pour ce qui donne le bonheur de vivre. Tu aurais effleuré de tes mains les boucles blondes des petits des hommes. Tu aurais puisé une joie douce, en plongeant longuement ton regard alangui de tendresse tout au fond de leurs yeux purs (...). Mais les mauvais hommes n'ont pas permis cela. Ils ont voulu que la terrible prison de la Haine s'infiltrât dans ton cœur. Alors tu t'es dressée, farouche, contre les lois iniques et contre les monstres qui les avaient faites (...). Soudain, poussée par une force invincible, tu as crié aux esclaves que l'heure de la révolte était enfin venue. » ( $L$ 2.03.1923)

Germaine Berton est ainsi présentée comme un modèle de militantisme et d'abnégation pour les anarchistes. Au confort et à la tranquillité quotidienne - ambitions considérées comme bourgeoises elle a fait le choix du risque et de l'action, renonçant à la vie proposée aux femmes dans la France des années vingt. Néanmoins, Le Libertaire n'accule pas les femmes à la révolte contre leur condition, mais les enferme dans les stéréotypes féminins. Mieux, il publie un appel non pas aux militantes mais « aux femmes» : 
"O femmes! O mères! qui chaque jour buvez vos larmes et que mord cruellement, chaque jour, le regret du petit tué par la boucherie, la famine ou la peste. N'oubliez pas Germaine Berton. Elle vous a donné ses vingt ans pour détruire un organisateur conscient, méthodique, implacable de la dernière guerre et de la prochaine. Les feuilles publiques dénaturent sa vie. Les clichés d'anthropométrie défigurent son visage. Ne retenez que sa jeunesse, son courage et sa foi. Si elle sort vivante de l'hôpital', défendez-la. Demander sa grâce, c'est vouloir la vôtre; la sauver, c'est vous sauver!" (26.01.1923)

La dimension politique du geste de Germaine Berton se trouve ainsi atténuée par le don de soi, vertu célébrée comme essentielle de la "nature féminine ». Si elle est présentée comme un exemple pour les femmes, ce n'est pas en terme d'émancipation et d'action comme le suggèrent les propos adressés aux "exploités », c'est-à-dire au monde ouvrier, au prolétariat, aux "esclaves », aux anarchistes. Les femmes ne sont pas ici considérées comme des esclaves à pousser à la révolte, mais comme des victimes, des êtres blessés dans leur chair par la guerre. La reconnaissance de Germaine Berton comme actrice politique à part entière, malgré la démonstration violente qu'elle s'autorise, se révèle fort délicate. Plus encore, les éléments permettant d'identifier la prise en considération de l'affirmation de la militante en tant que sujet politique " femme " sont rares. Elle n'a pourtant cessé de réaffirmer avec force le caractère éminemment politique de son crime.

Son crime est politique, certes, mais elle n'est pas considérée comme une individue actrice de la politique. La nuance est de taille et brouille en permanence les discours anarchistes. C'est là une limite importante des manifestations de solidarité dont Germaine Berton est l'objet.

\section{Une figure intégrée à l'histoire militante}

Les modes de légitimation de la violence se révèlent particulièrement intéressants. II s'agit d'une logique matérialiste, telle que la définit Isabelle Sommier (1998), logique « en vertu de laquelle l'usage de la violence est, somme toute, en conformité avec les lois objectives de l'Histoire et en continuité avec le mouvement révolutionnaire dont les groupes s'estiment les héritiers » (53). Deux analogies avec des événements historiques sont ainsi faites par Le Libertaire. Germaine Berton est ainsi comparée au militant anarchiste Louis-Émile Cottin qui a tenté d'assassiner Clemenceau en février 1919 :

"L'un et l'autre avaient vingt ans. Ils se sont sacrifiés pour toi, à cause de toi. L'un et l'autre aimaient la vie et exécraient le meurtre. Ils ont été vers la mort et ils ont tué, pour toi, à cause de toi, foule prolétarienne ! » (L 2.02.1923).

L'exaltation de la violence politique relève ici de la mise en parallèle avec les autres héros du mouvement anarchiste et Germaine Berton est masculinisée dans le propos à travers les références à I'honneur et au sacrifice. Pierre Naville (1982) explique en ce sens : « des femmes anarchistes ont

\footnotetext{
${ }^{6}$ Après avoir tiré sur Marius Plateau, Germaine Berton a retourné l'arme contre elle. Arrêtée sur le champ, elle est hospitalisée quelques jours.
} 
manié le revolver ou la bombe. Mais en presque tous les cas, leur rôle est apprécié ou exalté parce qu'il rivalise avec celui du héros mâle plutôt que par ses vertus propres » (129). Dans ce contexte s'opère un processus de masculinisation de ces femmes par le langage pour ce qui est du domaine de l'honneur et du sacrifice. Pour ce qui est de Germaine Berton, ce processus n'est pas permanent, et si Le Libertaire parle parfois d'elle comme d'un guerrier, la majeure partie du propos consiste cependant à la déterminer comme « femme ». Le caractère exceptionnel de son geste la virilise, mais ne doit pas masquer son identité féminine.

Mais c'est surtout le rapprochement avec l'assassinat de Jaurès qui domine. Et Germaine Berton l'affirme lors de son premier interrogatoire : elle a voulu venger Jean Jaurès, réparer l'injustice de l'acquittement de Villain, son meurtrier, et faire payer l'Action française, responsable. En conséquence, le procès sera celui des nationalistes pour l'Union anarchiste qui publie :

"Avec elle, nous ferons le procès de L'Action française et du Parti royaliste, et nous établirons la liste de leurs méfaits perpétrés depuis quelques dix ans (...). Quand Villain tua Jaurès, vous n'osâtes point le revendiquer après pourtant lui avoir armé le bras. Nous revendiquons, nous, l'héroïque Germaine Berton et pourtant ce n'est pas nous qui l'avons dressée contre vous, ce sont vos infâmes agissements » (L 26.01.1923).

Cette analogie avec le meurtre de Jaurès entraîne un intérêt et un regard particulier sur le geste de Germaine Berton, renforçant la dimension politique du crime pour ses partisans. Lors de son premier interrogatoire, Germaine Berton justifie ainsi son geste: « Ni la misère matérielle, ni la misère physiologique, n'ont influé sur mon acte dont je revendique la pleine responsabilité »(24.01.1923). S'adressant à l'audience à Léon Daudet, elle persiste : « J'ai voulu vous tuer parce que vous étiez responsable de l'assassinat de Jaurès. Je suis anarchiste, mais cela ne m'empêche pas de vénérer Jaurès " ( $L$ 20.12.1923). Si elle a cherché à venger Jaurès, elle n'a surtout, selon ses soutiens, fait que répondre aux provocations permanentes des nationalistes. Ce mode de légitimation est relativement classique dans l'histoire de la violence politique. Lors du procès, les témoins de la défense vont d'ailleurs s'appliquer à faire porter la responsabilité du meurtre de Plateau sur les épaules des nationalistes.

Néanmoins, des réserves sont apportées au geste de Germaine Berton, beaucoup de militants d'extrême gauche n'étant pas partisans de l'acte individuel, simple expression de la révolte, mais de l'organisation collective de la révolution: "Ennemis de la violence contre l'individu, nous ne l'érigerons jamais en système. Ce que nous voulons, c'est la révolte collective » (L 2.02.1923). On aurait pu supposer que les compagnons de lutte de la jeune femme la soutiendraient bec et ongle. Mais il s'avère difficile de plébisciter l'acte de Germaine Berton et d'être en mesure de proposer simultanément un projet politique crédible. Le crime de Germaine Berton n'est pas condamné en tant que tel ; il n'est pas approuvé dans l'absolu. La nuance a son importance. Les contradictions relevées permettent d'expliquer l'empêtrement dans lequel se trouvent les anarchistes du point de vue des rapports sociaux de sexe. 


\section{Comprendre le verdict à la lumière des rapports sociaux de sexe}

La conclusion de l'affaire Germaine Berton apportée par la Justice peut avoir de quoi surprendre. En effet, dans la soirée du 24 décembre 1923, après un court délibéré d'une quinzaine de minutes, la Cour d'assises - composée, rappelons-le, uniquement d'hommes - prononce l'acquittement, suivant ainsi les plaidoiries de la défense. A quelle logique pénale peut répondre l'acquittement alors que les preuves matérielles sont irréfutables et que l'accusée assume sa culpabilité ? Comment la presse traite-t-elle ce verdict ? Et, au fond, que révèle cette décision du point de vue de l'appréciation de la violence politique perpétrée par une femme?

Reprenons le réquisitoire du Ministère public, dont la fonction consiste à requérir l'application des lois au nom de la société, fonction qui enjoint donc une neutralité politique. L'avocat général Sens-Olive s'attache, dans un premier temps, à dresser un portrait de Germaine Berton emprunt de moralisme, détaillant, un à un, les traits de caractère condamnables de la jeune femme. Paresseuse, méchante, cruelle, hypocrite, Germaine Berton a agi seule, sans complice identifié, «l'exaltation de son esprit suffit à tout expliquer » (cité par Le Petit Parisien, 25.12.1923). Ensuite, il ne reconnaît aucune dimension politique à l'assassinat de Marius Plateau: « C'est un crime vulgaire, un crime banal » (cité par Le Petit Parisien, 25.12.1923) affirme-t-il. Cette position s'explique par les obligations de la fonction d'avocat général : pas de prise de position politique, mais un propos moralisateur et rigoriste.

Le verdict prononcé apparaît clairement s'inscrire dans une volonté d'apaisement des tensions nées de l'assassinat de Jean Jaurès. $M^{e}$ Henry Torrès, avocat des causes progressistes, plaidant l'acquittement de sa cliente, s'est attaché à mettre sur un même pied Raoul Villain. Un parallèle avec le meurtrier de Jaurès est tracé en raison de l'acquittement de ce dernier en mars 1919. Mais le sens de l'acquittement peut paraître surprenant. En effet, $M^{e}$ Torrès rejette toute idée de gloire pour Germaine Berton, alors que les témoins qu'il a faits citer se sont attachés, tout au long du procès, à saluer la bravoure et le courage de la militante anarchiste, quand ils n'enfonçaient pas les stéréotypes de la féminité. $\mathrm{M}^{\mathrm{e}}$ Torrès joue davantage sur la comparaison avec Villain que sur le fait que Germaine Berton soit une femme. Interviewé par Le Libertaire, il ne cache cependant pas que sa jeunesse et son sexe sont des éléments déterminants pour la juger : " elle est quand même une enfant de vingt ans, et depuis, souvent, est apparue la jeune fille avec ses naïvetés et ses élans sous la justicière impitoyable» (18.12.1923). II s'attache aussi à démontrer la responsabilité de l'Action française dans l'amplification de la violence, expliquant qu'il compte faire «le procès des violences réactionnaires, par lesquelles on voudrait incliner notre peuple, celui de 1793 et de la Commune, à un fascisme démarqué » ( L 18.12.1923). Pour lui, l'acquittement doit être pensé en ce sens ; il s'agirait d'un signal fort d'apaisement de la part d'une Justice qui n'a pas reconnu la culpabilité de l'assassin de Jaurès suivant ainsi la position de sa veuve, qui estimait que Villain n'était pas le véritable responsable. II faut alors renvoyer Germaine Berton d'où elle vient : l'anonymat. C'est en substance le contenu de sa plaidoirie : 
"Vous acquitterez Germaine Berton, que je ne glorifie pas : nulle gloire pour le sang versé. Vous l'acquitterez, pour qu'elle aille rejoindre Villain dans l'oubli et dans l'apaisement! " (cité dans Le Petit Parisien, 25.12.1923).

Torrès se démarque ainsi en partie de la rhétorique utilisée jusqu'au procès par Le Libertaire, n'exploitant pas le fait que l'accusée est une femme.

L'acquittement est salué par l'ensemble de la presse d'opinion qui a soutenu Germaine Berton comme une victoire pour la société du progrès et une condamnation indirecte des pratiques de l'Action française. Cette fois-ci, pas de propos quant au caractère bourgeois de l'institution judiciaire. A compter du verdict, les rapports de genre sont ignorés par Le Libertaire qui fait sienne la rhétorique de la défense. A ce niveau, Germaine Berton est enfin considérée comme anarchiste avant d'être femme. Elle ne devient pas une martyre; elle reste une héroïne intégrée au panthéon militant, mais de manière discrète puisqu'elle disparaît rapidement des figures emblématiques du mouvement anarchiste. En ce sens aussi, la mouvance anarchiste a suivi la position de $\mathrm{M}^{\mathrm{e}}$ Torrès puisque que Germaine Berton a aujourd'hui disparu de cette mémoire militante.

Les partisans de l'Action française accueillent le verdict comme une injustice, pire, une infamie. « Le crime du jury » (AF 25.12.1923) s'étale ainsi en une du quotidien nationaliste. L'acquittement est perçu comme l'ultime manifestation du complot orchestré contre l'Action française et de la compromission de la République ; le geste de Germaine Berton passe alors au second plan. Comme l'a remarqué Eugen Weber (1985), les nationalistes « aimèrent mieux rejeter cet acquittement sur l'injustice et la perfidie des républicains auxquels ils avaient d'abord attribué le crime » (165). Ils en profitent donc pour rappeler leurs positions politiques et attaquer la République, la « femme sans tête », la « Gueuse » :

"Le cri est unanime : "Nous étions encore républicains... mais maintenant, ce n'est plus "Marianne", c'est "Germaine" qu'il faut appeler la République (...). A Germaine donc l'honneur du sanglant symbole, indiscutablement lié à l'histoire de la trahison, comme à celle de la démocratie toute pure » (AF 28.12.1923).

Plus encore, la République ne serait qu'une marionnette au service des «bolcheviques » : "Par la méchanceté des uns et la faiblesse des autres, la Révolution aux ordres de l'Allemagne vient de remporter un incontestable succès " (AF 26.12.1923). Le verdict permet ainsi à l'Action française de se positionner en victime d'un système corrompu par le parlementarisme, le capitalisme juif, le communisme, système sans cesse dénoncé dans les colonnes du journal.

En acquittant Germaine Berton, la Cour retire au geste sa valeur de révolte ; la dimension politique du crime s'en trouve altérée et la jeune femme ne sera pas la martyre qu'elle aurait vraisemblablement 
faite si une condamnation avait été prononcée ${ }^{7}$. Le procès que $M^{e}$ Torrès souhaitait intenter à l'Action française s'est ainsi tenu, comme il le souligne dans ses mémoires (1957) : « J'entendais transformer le procès de Germaine Berton en procès de l'Action française et je crois y être parvenu » (40). Ainsi, l'acquittement de Germaine Berton, qui peut paraître particulièrement surprenant du point de vue juridique, est prononcé. L'hebdomadaire L'lllustration propose une explication qui nous semble significative : alors que Germaine Berton revendique son geste, que les preuves matérielles chargent l'accusée, des circonstances atténuantes lui sont accordées à demi mot : "les douze jurés n'ont pas voulu, en rendant ainsi à la liberté la meurtrière du malheureux Marius Plateau, innocenter son geste, mais ils ont été sensibles aux circonstances qui atténuaient sa responsabilité : son enfance malheureuse, la misère, la griserie contagieuse des milieux anarchistes qu'elle fréquentait » (29.12.1923).

Seule une voix s'élève pour souligner l'effet pervers de l'acquittement, celle des surréalistes : acquitter Germaine Berton, c'est vouloir étouffer la force de son geste. Louis Aragon compose ainsi un poème «Germaine Berton », véritable hymne à la révolte, publié en 1924 dans le premier numéro de La Révolution surréaliste. Ce rejet du compromis ne sera cependant pas repris par les anarchistes, pourtant enclins aux positions radicales.

\section{Conclusion}

Michelle Perrot (2001) avance un sens à l'acquittement d'une criminelle du point de vue des rapports sociaux de sexe : "le crime, le délit sont affaires d'hommes, actes virils commis dans la jungle des villes. Est-ce l'indice d'une soumission, d'une moralisation accrues de la femme que son effacement sur ce théâtre? Ou une certaine manière de la refouler en coulisses ? Cette indulgence, au fond, n'est-elle pas suspecte ? Refuser à la femme sa stature criminelle, n'est-ce pas encore une façon de la nier ? » (179). Le cas de Germaine Berton pose la question de déterminer de quelle façon un crime politique commis par une femme est traité, quelle place occupe le genre dans les discours et comment ceux-ci sont guidés par des stéréotypes et des peurs sociales.

II est frappant d'observer à quel point Germaine Berton fait l'objet d'un traitement résolument non politique alors que le crime est lui-même considéré comme politique. Lui retirer en partie l'initiative de son acte, considérer qu'elle n'a fait que répondre aux provocations de l'Action française, se livrer à de longues descriptions pour déterminer sa moralité, pour finalement l'acquitter, bien qu'elle affirme haut et fort le sens de son geste, tout cela est particulièrement révélateur de la stratégie de minimisation de sa capacité d'exister en tant que sujet politique. La violation des règles sexuées du militantisme, aussi radical soit-il, se trouve ici particulièrement sanctionnée. II n'est pas concevable, en 1923, de

\footnotetext{
${ }^{7} \mathrm{M}^{\mathrm{e}}$ Torrès fait part de cette crainte dans ses mémoires (1957) : « Je savais qu'elle ambitionnait d'être condamnée à une forte peine. "Germaine Berton, vous avez trois jours francs pour vous pourvoir en cassation. Passé ce délai, vous n'y serez plus recevable». Sur ces paroles du président, elle se serait levée, bien droite dans son box et, au cri de "Vive l'anarchie !", se serait poignardée à l'antique», p.39.
} 
considérer une femme qui transgresse violemment l'ordre social et sexué autrement qu'à la lumière du déterminisme biologique. Aux habituelles circonstances atténuantes accordées aux femmes, la Justice a ajouté un acquittement particulièrement surprenant du point de vue pénal. Dans son analyse de la construction de la virilité, George Mosse (1997) observe les réactions de la société face à celles que l'on qualifie de garçonnes : « une fois de plus, la société s'arrangea pour assimiler les éléments nouveaux et perturbateurs afin de pouvoir les contrôler. Ce mécanisme s'appuyait sur un consensus qui pénétra les sensibilités politiques les plus opposées, de droite comme de gauche » (169). Force est de constater que le mécanisme a été passablement identique pour Germaine Berton. Ainsi, en ne la condamnant pas, le mécanisme d'assimilation a évité d'en faire un modèle pour les anarchistes dont elle a disparu de la mémoire militante - et pour les femmes, leur démontrant que l'usage de la violence ne leur serait d'aucune aide. "Car la femme qui prend les armes, quelle que soit la justesse de sa cause, ne peut que mal finir, et on ne l'excuse de le faire que si c'est contre son gré (Jeanne, la douce bergère obéissant à Dieu et à ses voix) et si elle finit mal. La guerrière n'est justifiée que par son sacrifice qui lui fait réintégrer le rôle de victime dévolu aux femmes, et par une vie irréprochable qui lui permet d'accéder à la sainteté. II fallait que Jeanne soit réputée pucelle pour pouvoir passer à la légende de l'Histoire »(Le Garrec, 1987: 87). Germaine Berton est sortie de la bienséance féminine, elle a pris l'initiative de tuer un opposant politique et la dimension sacrificielle de son geste a été gommée par son acquittement. De ce point de vue, Germaine Berton n'a rien eu pour elle.

\section{Bibliographie}

Apfelbaum, Erika (2004 [1 $1^{\text {re }}$ éd. 2000]). « Domination». In Helena Hirata et al. (Ed.), Dictionnaire critique du féminisme (pp. 44-49). Paris : PUF.

Bard, Christine et Françoise Thébaud (1999). "Les effets antiféministes de la Grande Guerre ». In Christine Bard (Ed.), Un siècle d'antiféminisme (pp.149-166). Paris : Fayard.

Burnet-Vigniel, Marie-Claude (1990). "Femmes russes dans le combat révolutionnaire. L'image et son modèle à la fin du XIX siècle ». Cultures et sociétés de l'Est, 12

Chauvaud, Frédéric (2002). "Introduction à la quatrième partie ». In Christine Bard et al. (Ed.), Femmes et justice pénale, $X I X^{e}-X X^{e}$ siècles (pp. 275-282). Rennes : PUR.

Guillaumin, Colette (1992). Sexe, race et pratique du pouvoir. L'idée de nature. Paris : Côté-femmes.

Le Garrec, Evelyne (1978). «Postface ». In Françoise d'Eaubonne, Contre-violence ou la résistance à l'État. Paris : Tierce.

Mosse, George L. (1997). L'image de l'homme. L'invention de la virilité moderne. Traduit de l'américain, Paris : Abbeville/Pocket.

Naville, Pierre (1982). « La femme soldat ». Pénélope, 6, 129-133.

Perrot, Michelle (2001). Les ombres de l'histoire. Crimes et châtiment au XIX siècle. Paris: Flammarion.

Sommier, Isabelle (1998). La violence politique et son deuil. L'après 68 en France et en Italie. Rennes : PUR. 
Torrès, Henri (1957). Accusés hors série, Paris : Galllimard.

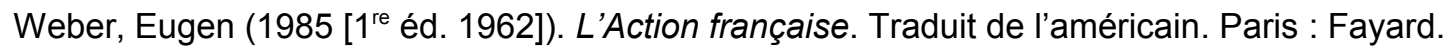

